\title{
Jupiter Moon May Hold Hidden Sea
}

The roof is leaking but we don't mind, we're glad for the sound, the plip of water

filling the pot we've laid on the floor.

On that first night we took a boat across a harbor.

It must have been an effort, keeping track of me in the dark.

And in the morning, sunlight through the east window, the air still cool.

How many times will one person imagine light shining through one small east-facing window?

More than I would have imagined.

Each day something makes us walk out

to the sandbars and later say I walked out to the sandbars, I put my foot down on a shore. 